\title{
Reflets
}

Revue ontaroise d'intervention sociale et communautaire

\section{Précarisation socioprofessionnelle : trajectoires de jeunes travailleurs devenus toxicomanes}

\section{Pauline Morissette, Marie-France Maranda et Danielle Lessard}

Volume 14, numéro 1, 2008

Travail, jeunesse et intervention

\section{URI : https://id.erudit.org/iderudit/018854ar}

Aller au sommaire du numéro

Éditeur(s)

Reflets : Revue d'intervention sociale et communautaire

ISSN

1203-4576 (imprimé)

1712-8498 (numérique)

Découvrir la revue

Citer cet article

Morissette, P., Maranda, M.-F. \& Lessard, D. (2008). Précarisation

socioprofessionnelle : trajectoires de jeunes travailleurs devenus toxicomanes. Reflets, 14(1), 38-65.
Résumé de l'article

L'insertion professionnelle des personnes toxicomanes pose un défi particulier pour les milieux de la réadaptation en toxicomanie et pour les services spécialisés en matière d'emploi. Peu d'études ont mis en évidence des processus de précarisation du travail chez les jeunes toxicomanes. Pour ce faire, nous avons privilégié le récit de vie comme démarche de recherche. L'objectif du présent article est de montrer des dynamiques qui sous-tendent les trajectoires socioprofessionnelles de quinze jeunes consommateurs âgés de 18 à 30 ans. Quatre dynamiques sont présentées, illustrées et concrétisées par un récit.
Tous droits réservés @ Reflets : Revue d'intervention sociale et communautaire, 2008 services d’Érudit (y compris la reproduction) est assujettie à sa politique d'utilisation que vous pouvez consulter en ligne.

https://apropos.erudit.org/fr/usagers/politique-dutilisation/ 


\section{Précarisation socioprofessionnelle : trajectoires de jeunes travailleurs devenus toxicomanes $^{12}$}

"...la recherche dont il sera question dans cet article cherche à comprendre les dynamiques de précarisation des trajectoires socioprofessionnelles de jeunes toxicomanes de 18 à 30 ans. »

\author{
Pauline Morissette \\ Professeure titulaire, École de service social, Université de Montréal \\ Marie-France Maranda \\ Professeure titulaire, Université Laval \\ Danielle Lessard \\ Université de Montréal
}

L'insertion professionnelle des personnes ayant vécu une expérience de toxicomanie pose un défi particulier pour les milieux de la réadaptation en toxicomanie et pour les services spécialisés en matière d'emploi. Des données d'enquêtes américaines, québécoises et européennes et des données cliniques décrivent les toxicomanes comme un groupe particulièrement vulnérable sur le plan de l'emploi (OFDT, 2002; Bray et collab., 2000; Platt, 1997). Les transformations structurelles du monde du travail pourraient exercer encore davantage de pressions sur cette population (Iversen, 1998). En lien avec ces transformations, la recherche dont il sera question dans cet article cherche à comprendre les dynamiques de précarisation des trajectoires socioprofessionnelles de jeunes toxicomanes de 18 à 30 ans. L'attention a porté sur les enchaînements d'éléments familiaux, scolaires et professionnels qui les ont menés à des situations socioprofessionnelles précarisées (c.-à-d. emploi précaire, sans emploi ou dans des emplois syndiqués, mais menacés de perte 
de leur emploi pour des raisons de consommation). Le terme toxicomane réfêre à un consommateur abusif de substances psychoactives : alcool, drogues illégales ou les deux.

\section{Réalités du marché du travail et difficultés d'insertion professionnelle}

Ces dernières décennies, les nouvelles règles régissant les marchés économiques ont entraîné des changements organisationnels. Cette situation a conduit les entreprises à adopter des stratégies de flexibilité qui ont contribué à l'accroissement du chômage dans certains pays (p. ex. la France) et ont mené à la création d'emplois appelés précaires (p. ex. le Québec et le Canada). Ce type d'emplois précaires ou atypiques est caractérisé par une durée incertaine ou limitée, dont le statut ne donne aucun droit à des avantages sociaux ou à des régimes de retraite (Bernier et collab., 2003; Cranford, Vosko et Zukewich, 2003; Howard, 1995). La flexibilité a aussi été associée à des changements dans les relations de travail, dans la nature du travail proposé et dans l'organisation sociale en général (Purcell et Purcell, 1998; Pollert, 1988). Désormais la précarité de l'emploi et du travail tend à être reconnue comme une situation qui se généralise et se normalise dans l'organisation du travail (Castel, 2007). Or, l'importance de l'insertion professionnelle comme un pas dans le processus de sortie de la toxicomanie (Dambuyant-Wargny, 2006; Jamoulle, 2000; Castel, 1998), s'avère cruciale autant pour les personnes concernées que pour la société.

Plusieurs auteurs s'entendent toutefois pour dire que les jeunes sont particulièrement affectés par la montée du chômage ou l'émergence des nouvelles formes d'emplois précaires (Rose, 2000 et 1998), notamment les jeunes aux prises avec la toxicomanie. Jusqu'ici, des enquêtes menées dans des centres de réadaptation, aux États-Unis, en France et au Québec montrent une forte proportion d'inactivité professionnelle chez la jeune clientèle 
toxicomane (Lopez, 2002; Platt, 1997; Landry, Guyon et Beauvais, 1997) et l'alternance d'emplois précaires, situations de chômage et assistance sociale chez les jeunes consommateurs (Morissette et Boivin, 1999).

Des études cliniques, épidémiologiques et ethnographiques effectuées aux États-Unis, en France, en Belgique et au Québec indiquent aussi qu'ils sont nettement plus désavantagés socialement que bien des jeunes de leur âge en raison de l'absence de diplôme, de la présence d'un dossier judiciaire ou d'une vulnérabilité émotionnelle (Jamoulle, 2000; Mathis et collab., 1994; Facy et Le Huede 1992). Une situation, qui, à elle seule, pourrait entrainer leur éviction du marché du travail ou restreindre leur accès à des emplois intéressants. Les écrits indiquent aussi que leur mode de vie ou leur consommation pourrait les rendre vulnérables à la sélection opérée par les employeurs (Roman et Blum, 1999) et qu'ils sont souvent considérés comme des "travailleurs à risque " (Cook, 2002), malgré certaines compétences.

Outre ces données générales ayant trait à la condition de jeunes toxicomanes travailleurs et à leur degré d'intégration à la vie active, plusieurs recherches ont mis l'accent sur les prédicteurs et les conséquences de l'usage de substances psychoactives parmi les employés. Même si, d'après Frone (2003) peu de chercheurs se sont arrêtés aux jeunes employés ou aux conditions de travail pouvant les amener à faire usage d'alcool ou de drogues, la littérature permet de classer les prédicteurs en deux perspectives générales : celle qui envisage les causes de l'usage de substances, comme externes au milieu de travail, et celle qui les voit d'une perspective interne. Selon Frone (2003), la perspective externe renvoie à des prédispositions personnelles des employés à consommer et à leur incapacité à résister aux produits. La perspective interne réferre plutôt à des mécanismes de contrôle social dans les milieux

"Peu d'études ont jusqu'ici mis en évidence des processus de précarisation $d u$ travail chez les jeunes toxicomanes. » de travail, par exemple les politiques formelles ou informelles de consommation, la disponibilité du produit et les normes de consommation (Ames, Grube et Moore, 2000) et au stress du milieu de travail (Frone, 1999).

Peu d'études ont jusqu'ici mis en évidence des processus de précarisation du travail chez les jeunes toxicomanes. La 
précarisation est définie par Appay (2005, p. 49) comme étant "Un processus de restructuration productive et de fragmentation s'accompagnant d'une déconstruction des droits sociaux et portant atteinte à la santé publique, dont l'aboutissement est l'individualisation du travail et la déconstruction des contrepouvoirs au travail ». Comme le fait remarquer Saint-Martin (2007) en parlant de la précarisation des populations urbaines et de la fragilisation professionnelle, la précarisation est un phénomène social qui fait état d'un double processus : «l'un, de la précarisation économique qui résulte de la précarisation salariale et de celle des structures productives; l'autre, d'institutionnalisation de l'instabilité à travers les transformations des systèmes législatifs afférents au travail et à la protection sociale " (Appay et Thébaud-Mony, 1997, p. 520). C'est le cas de ces jeunes travailleurs devenus toxicomanes, interviewés dans le cadre de cette recherche, qui ont connu des trajectoires de vie personnelle et économique marquées par cette double précarisation à la fois économique et personnelle. L'objectif spécifique de cet article est de montrer les dynamiques sous-jacentes à ce processus de précarisation. Ces dynamiques ne prétendent pas viser l'exhaustivité et la représentativité, mais dégager plutôt le sens de certaines trajectoires socioprofessionnelles que l'on retrouve chez les jeunes toxicomanes.

\section{Méthodologie}

La démarche de recherche privilégiée est le récit de vie recueilli lors de plusieurs rencontres (Legrand, 1993). Les récits de vie permettent de repérer les dimensions objectives et subjectives de l'expérience du travail des jeunes consommateurs interviewés et de saisir le sens de leur expérience à partir de leur histoire individuelle, familiale, sociale et professionnelle. Ils permettent aussi de comprendre leur trajectoire socioprofessionnelle et la dynamique qui les sous-tend. Les trajectoires sont décrites comme une succession d'événements objectifs et subjectifs (Bertaux, 1997) ayant mené les jeunes consommateurs vers des situations 
précarisées de travail. Chaque trajectoire est marquée d'une dynamique particulière, c'est-à-dire de l'ensemble des éléments intervenant «en réseau » ou simultanément dans une histoire (Mucchielli, 1996).

Le modèle d'analyse présenté ci-après est un soutien à la compréhension des résultats et non un modèle explicatif. Nous schématisons en ordonnée et en abscisse quatre pôles pour l'analyse de la précarisation: les déterminants contextuels et individuels, les stratégies de défense et d'action.

Figure 1

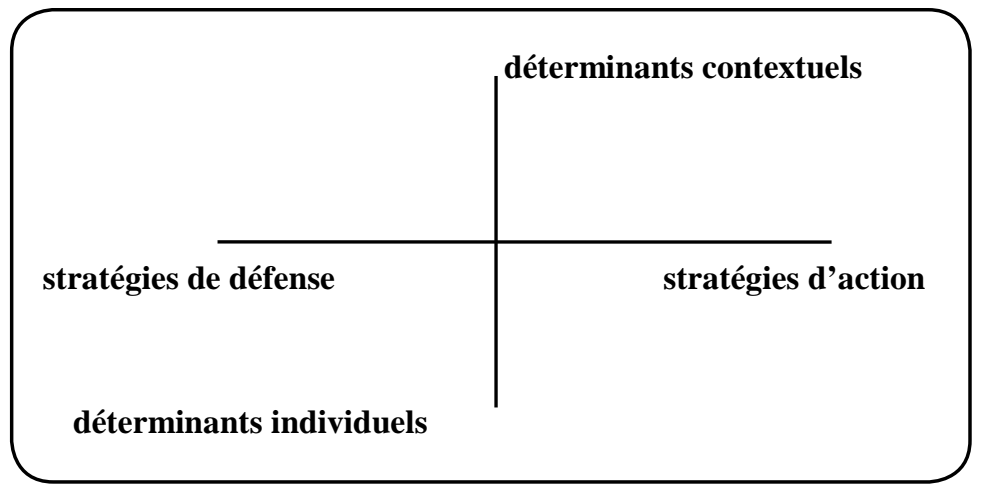

Les déterminants contextuels englobent les contraintes et les normes du marché du travail, les lois reliées aux différentes structures ou organisations de la société (p. ex. le contexte du marché du travail, les emplois disponibles ou non disponibles, le contexte gouvernemental, l'organisation du travail, la gestion des ressources humaines, les conditions de travail, les exigences du travail ou de l'école). Les déterminants individuels réferent au capital humain :le niveau de scolarité, le diplôme, les expériences de travail, l'état de santé, les compétences professionnelles; et au capital social : la famille et le réseau relationnel du jeune travailleur. Sa personnalité, ses valeurs ou ses motivations, ses capacités d'adaptation complètent le tableau. L'axe horizontal comprend, d'une part, les stratégies de défense et, d'autre part, les stratégies d'action ou d'adaptation de la personne. Les stratégies 
"Les trajectoires ont été construites sous l'effet conjugué des différents "pôles " qui sont liés de multiples façons, reflétant les interactions et la nature dynamique de la précarité et $d u$ processus de précarisation. » de défense sont construites afin de contrer les situations difficiles et de transformer la perception d'une réalité agressante. Le recours aux substances psychoactives pour atténuer ou fuir une réalité pénible du travail en est un bon exemple. Les stratégies d'action ramènent à l'ensemble des moyens déployés par les jeunes consommateurs désireux de s'insérer sur le marché de l'emploi, par exemple les scolarités à compléter, les orientations et réorientations professionnelles et le développement de réseaux d'embauche. Les trajectoires ont été construites sous l'effet conjugué des différents "pôles " qui sont liés de multiples façons, reflétant les interactions et la nature dynamique de la précarité et du processus de précarisation.

\section{Échantillon}

Les participants de l'étude étaient âgés de 18 à 30 ans et répondaient à une définition de consommation abusive de drogues et/ou d'alcool dans l'année précédant l'enquête. Les tests utilisés sont le DAST-20 pour la consommation de drogues (Skinner, 1982) et le SMAST pour la consommation d'alcool.Tous avaient un score supérieur à 11 au DAST (un niveau de consommation de drogues de substantiel à sévère) ou supérieur à 18 au SMAST (une consommation abusive d'alcool). Quatre participants ont obtenu des niveaux sévères de consommation aux deux tests.

Les participants ont été recrutés dans différents milieux: un centre de réadaptation pour alcooliques toxicomanes, un organisme d'insertion à l'emploi, une entreprise de réinsertion et diverses grandes entreprises. Géographiquement, ils habitaient les régions métropolitaines de Montréal et de Québec.

Notre corpus d'entretiens comprend une trentaine d'entrevues d'une heure et demie en moyenne, menées auprès de 15 participants; nous les avons rencontrés au minimum deux fois chacun. Dix interviewés étaient des hommes et cinq étaient des femmes. Une majorité avait plus de 25 ans. Tous étaient dans une situation socioprofessionnelle de précarité : emploi précaire, sans emploi ou dans des emplois syndiqués, mais menacés de perte d'emploi pour des raisons de consommation. 


\section{Collecte des données et construction des trajectoires de précarisation}

Les entrevues effectuées ont été espacées d'au plus trois semaines pour permettre une interanalyse entre les trois chercheuses. $\mathrm{La}$ première rencontre débutait par une question très générale pour amener les participants à raconter et à décrire leur histoire de travail. Au cours de cette première rencontre, les participants décrivaient chacun des emplois occupés : type d'entreprises, statut et liens d'emplois, présence ou non d'un syndicat. Ils discutaient aussi des motifs de plaisir ou de déplaisir à occuper certains emplois. Après chaque entretien, le contenu intégral était retranscrit et un bilan était réalisé. Ce bilan permettait de suivre l'évolution de la trajectoire professionnelle et personnelle et de mettre en lumière des moments-clefs qui traversent l'histoire du participant. Chaque bilan était par la suite retranscrit dans une grille élaborée pour cette recherche. Cette grille illustrait l'essentiel de sa première entrevue. Lors de la deuxième entrevue, la grille était présentée au participant pour démarrer la discussion. Le répondant était invité à vérifier notre compréhension (nos hypothèses) de son histoire, à lui apporter des précisions ou à la rectifier si nécessaire. À la suite de la deuxième entrevue, les étapes d'analyse ont été les suivantes :

"L'approche

d'analyse de base pour la construction de notre typologie s'inspire des principes de la théorisation ancrée (Strauss et Corbin, 1998), dans le sens qu'elle est enracinée dans les données et émane d'une comparaison constante."
1) D'abord, une analyse verticale des deux entrevues a été effectuée pour chaque participant. Une autre grille a été alors construite pour épurer, gérer et organiser de façon systématique les données des deux entrevues. Cette façon de procéder a permis de visualiser graphiquement les parcours généraux de l'ensemble des participants, de faire ressortir les points saillants des parcours et ainsi de faire émerger des sousgroupes ayant des parcours individuels ou sociostructurels semblables.

2) Ensuite, une analyse comparative intragroupe a fait ressortir les structures communes.

3) Finalement, une analyse comparative intergroupe a permis de mettre en perspective les catégorisations générales et les dynamiques. L'approche d'analyse de base pour la construction 
de notre typologie s'inspire des principes de la théorisation ancrée (Strauss et Corbin, 1998), dans le sens qu'elle est enracinée dans les données et émane d'une comparaison constante.

\section{Résultats}

"L'entrée dans la consommation leur a permis de supporter leur vie et un quotidien trop douloureux (p. ex. violence familiale, violence sexuelle et inceste). "
Les quatre trajectoires de précarisation socioprofessionnelles des jeunes toxicomanes sont d'abord expliquées théoriquement et illustrées à l'aide d'une figure. Ensuite, un récit vient concrétiser et humaniser les notions théoriques.

\section{Première trajectoire : une dynamique d'infériorisation}

La première trajectoire de précarisation, telle qu'illustrée dans la figure qui suit, présente des jeunes toxicomanes qui ont cumulé depuis l'enfance de nombreuses expériences de domination et d'exploitation. Celles-ci ont été vécues dans différentes sphères de leur vie (milieux familial, scolaire et professionnel) et à divers moments de leur histoire. Toutes ont eu des effets d'infériorisation.

Lâge du début de la consommation est un premier élément de précarisation qui a été observé dans cette trajectoire. En effet, ces jeunes ont commencé à consommer de l'alcool et des drogues vers l'âge de 11 ou 12 ans. L'entrée dans la consommation leur a permis de supporter leur vie et un quotidien trop douloureux (p. ex. violence familiale, violence sexuelle et inceste). Ces expériences ont très tôt provoqué chez les jeunes de cette trajectoire un sentiment d'infériorité sociale (De Gaulejac, 1999), c'est-à-dire un sentiment de ne pas être, selon leurs mots, "comme il faut". La majorité a été placée dans des familles ou des foyers d'accueil. Ces déplacements auraient continué à miner leur confiance et à modifier leur vision d'eux-mêmes et de leur avenir. Dès leur entrée sur le marché du travail, la drogue aurait permis de rendre "tolérables " des situations par ailleurs intolérables. La 
plupart des jeunes toxicomanes de cette trajectoire ont occupé des emplois qui, selon eux, étaient " abaissants et humiliants " en raison d'exigences particulières ou du fait qu'ils les considéraient socialement dévalorisés. Il s'agissait d'occupations telles qu'ouvrier agricole, serveuse-danseuse, déménageur. Ils ont aussi révélé que dans ce type d'emplois, la violence (harcèlement, violence physique ou verbale) était monnaie courante et partie intégrante de l'exercice du métier ou des rapports qu'ils avaient avec les employeurs. Ces formes de violence leur rappelaient celles vécues dans leur famille.

Figure 2

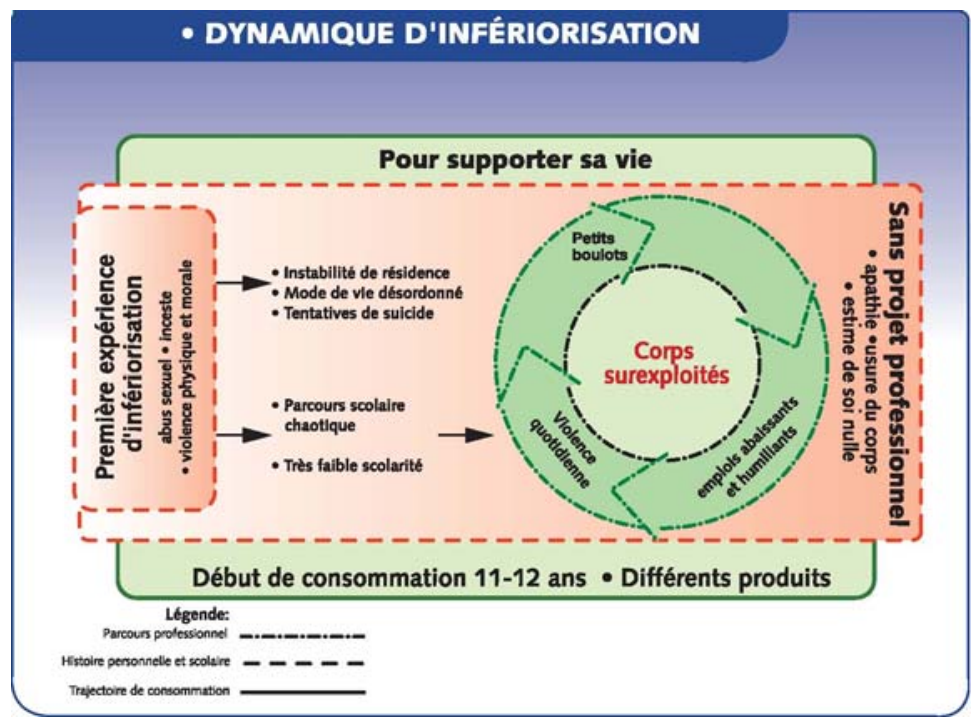

Ces jeunes sont sans diplôme. Malgré leur capacité intellectuelle de poursuivre des études, ils ont décroché très tôt du système scolaire. Ils ont révélé ne pas avoir eu l'encadrement familial nécessaire pour fréquenter assidûment l'école. Autrement dit, ils ont manqué de soutien familial à un moment stratégique pour leur avenir et c'est là un maillon important des enchaînements négatifs subséquents. Selon les chercheurs en insertion socioprofessionnelle (Laflamme, 2000), cette absence de diplôme aggraverait les autres 
éléments infériorisants de cette trajectoire. En effet, le diplôme renvoie aux qualités intellectuelles des individus et permet aux employeurs de juger de leurs capacités de travail. A contrario, son absence conduit vers la marge et la marginalisation.

Par la suite, leur faible scolarité les a confinés dans des petits boulots non qualifiés, sans protection sociale, mal payés et dans lesquels on est généralement congédié sans préavis (p. ex., travail de manutentionnaire dans les entrepôts). On reconnaît dans leur parcours professionnel le mouvement nommé par Rose (2000) " appel-rejet de la main-d'œuvre " qui illustre une demande de flexibilité qui est surtout fonction des besoins spécifiques des employeurs ou des clients. Ces contextes de travail ont aussi nourri et aggravé leur impression d'être socialement inférieurs.

Au fil des ans, les jeunes de cette trajectoire se sont retrouvés avec un corps usé prématurément. Ils étaient conscients qu'étant sans diplôme, ils avaient leur corps comme principale ressource à utiliser sur le marché du travail, comme objet de travail ou outil de travail (Dambuyant-Wargny, 2006). Comme l'explique Dambuyant-Wargny (2000), ils savaient que leur corps surexploité et surutilisé est le dernier capital qu'ils ont à offrir aux employeurs. À l'analyse, on comprend que peu à peu leur capital physique a aussi été altéré par un mode de vie désordonné, combiné à plusieurs années de consommation. Les tentatives de suicide révélées par certains sont un autre témoignage de la profondeur de la souffrance qui a traversé leur vie.

Quand ils abordent la question de leur projet professionnel, les jeunes toxicomanes de cette trajectoire démontrent une apathie remarquable, c'est-à-dire une désillusion complète vis-à-vis des possibilités de réussite. Ils évoquent un sentiment de piétinement, une impression d'enfermement et de spirale reproductive sans fin par rapport à l'emploi.

\section{Mélanie, un mauvais départ...}

Je n'ai pas eu une belle enfance. J'ai grandi avec ma mère et ma grande sœur. Mon père, je ne l'ai pas beaucoup connu parce qu'il ne s'est jamais séparé de sa femme. J'ai six demi-frères et demi-sours. Ils savent que 
j'existe, mais ils ont toujours fait comme si je n'existais pas. Ma mère avait bien des problèmes. Elle me faisait garder à gauche et à droite, souvent par du monde que je ne connaissais presque pas, jamais elle ne m'a demandé si j'aimais ça. À sept ans, un ami de la famille m'a agressée. À 12 ans, ça s'est reproduit avec celui que je commençais à considérer comme mon beau-père, parce que ma mère et lui étaient pour se marier. Ma mère n'a rien fait pour empêcher cela. Lui, il me faisait travailler dans son dépanneur, il en profitait pour me toucher. C'est à peu près à ce moment-là que j'ai commencé à fumer du cannabis avec ma meilleure amie. J'aimais ça parce que j'oubliais mes problèmes de famille. Là,je ne pensais à rien. La drogue a toujours en cet effet sur moi. Bien gelée, je pouvais endurer bien des affaires. À 13 ans, je suis partie vivre avec ma sour. Là, je faisais ce que je voulais. J'allais dans les bars puis des hommes me payaient de l'alcool, parfois de la mescaline et de la cocaïne.

À 15 ans, j'ai réussi à terminer mon secondaire I. J'ai essayé de continuer à étudier, mais à chaque fois que j'avais une peine d'amour, $j$ 'arrêtais. Alors maintenant, $j$ 'ai juste quelques cours qui sont complétés dans chaque année.

C'est plus entre 15 ans et 17 ans que j'ai eu mes premières expériences de travail, mais c'était des emplois à temps partiel parce que j'étais encore à l'école. À 17 ans, j'ai laissé l'école et j'ai commencé à danser dans les bars. Là, ma consommation a augmenté, je buvais puis je prenais de la cocaïne, de l'acide, des drogues de rave, du cannabis. J'ai fait aussi deux overdoses. Je pense que je voulais disparaître à ce moment-là. Quand j'étais tannée de danser, je me trouvais des emplois de vendeuse ou de serveuse, mais j'ai toujours été renvoyée, je ne faisais jamais l'affaire. Ils disaient que je ne travaillais pas assez vite. Une fois j'ai été renvoyée parce que j'ai refusé de me mettre à moitié nue comme serveuse. Ce qui arrivait aussi c'était que les employeurs engageaient plein de monde juste pour le temps des fêtes, mettons, novembre, décembre. Après ça, ils les mettaient à la porte. C'était toujours des emplois où ils gardaient les meilleurs, les plus beaux, les plus fins.

Là,j'ai 25 ans et je suis en cure de désintoxication pour la troisième fois. Ce que je veux, c'est de me trouver un emploi où je pourrais arrêter de danser et de me prostituer. Mais je n'ai pas d'illusions, je n'ai pas de diplôme, pas d'expérience ou, en tout cas, pas beaucoup et j'ai toutes sortes de problèmes de santé, mon foie, mes dents, un herpès. Je prends 
deux médicaments pour soigner ma dépression. Avec tout ça, je ne sais pas encore comment gagner ma vie.

"La dynamique d'infériorisation qui sous-tend cette trajectoire est fonction d'un double effet : structurel (emplois socialement dévalorisés et précaires, mode de gestion "sélective") et individuel (absence de diplôme qualifiant, expériences familiales dégradantes). "

"Se sentir différents, associé à la peur du rejet, les a ensuite suivis jusque dans les milieux de travail."
La dynamique d'infériorisation qui sous-tend cette trajectoire est fonction d'un double effet : structurel (emplois socialement dévalorisés et précaires, mode de gestion "sélective ») et individuel (absence de diplôme qualifiant, expériences familiales dégradantes). On y retrouve donc un amalgame de facteurs de précarisation qui se sont enchaînés, croisés et renforcés.

\section{Deuxième trajectoire : une dynamique d'identification}

Dans le cas de cette trajectoire, nous avons noté un enchaînement négatif de situations personnelles et un processus de détérioration de la situation professionnelle de ces jeunes parallèlement à l'aggravation de leur consommation. Le rejet qu'ils ont expérimenté dans leur enfance en raison de caractéristiques particulières : " cheveux roux, obésité, malformations physiques, comportements dérangeants (cleptomanie) " est un élément marquant de leur vie. Ils ont intégré un sentiment d'être différents dès leur entrée à l'école, tel qu'illustré à la figure 3. Se sentir différents, associé à la peur du rejet, les a ensuite suivis jusque dans les milieux de travail. Tous ces jeunes toxicomanes se sont décrits comme des personnes renfermées, timides et complexées.

Paradoxalement, la consommation de substances psychoactives leur a servi de stratégies d'intégration dans des secteurs d'emploi (p. ex. la restauration) dans lesquels les produits sont disponibles et banalisés. Par exemple, l'alcool fait partie de "l'esprit maison " (Paugam, 2000). Lorsque les emplois sont répétitifs (p. ex., travail sur des chaines de montage), la consommation d'alcool (et même de drogues) représente une action collective pour se libérer des frustrations d'un quotidien professionnel étouffant. Dans ces cultures de travail, c'est aussi « pour faire partie de... » et pour être acceptés dans le groupe qu'ils ont commencé à consommer. Le milieu de travail a agi dans cette dynamique à titre de renforcement $\mathrm{du}$ fait que les pratiques de consommation en groupe constituent 
des moyens de socialisation. Dans ces types de milieux, les jeunes toxicomanes de cette trajectoire ont trouvé leur place. Ce contexte a contribué à l'enchaînement négatif qui a suivi, soit à l'augmentation de leur consommation et à la dégradation de leur situation d'emploi.

Figure 3

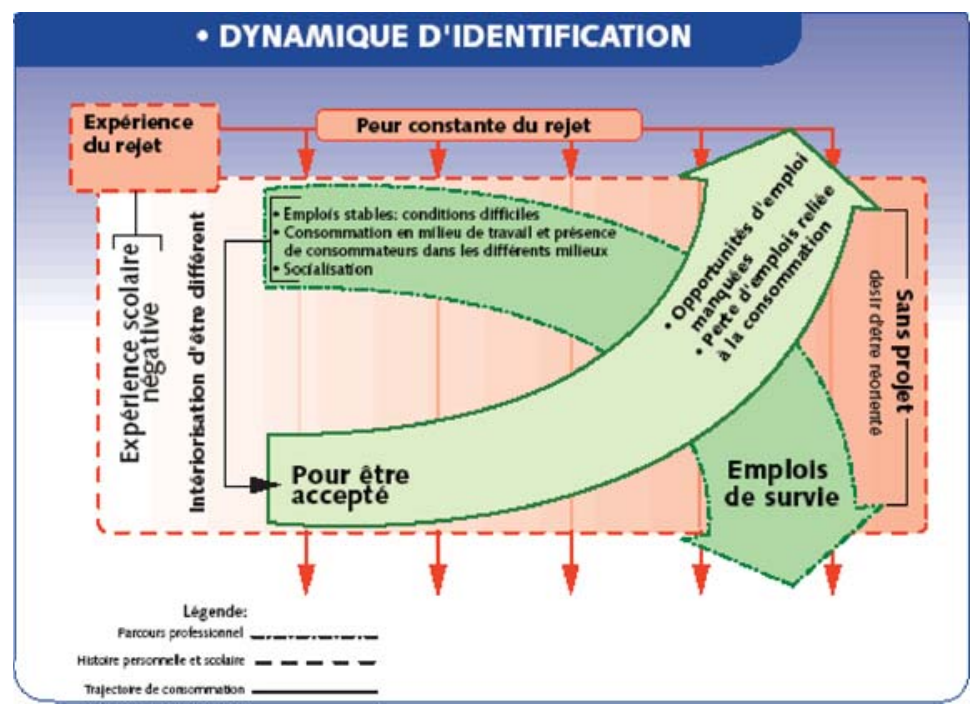

Néanmoins, ces jeunes consommateurs se sont unanimement dits plus à l'aise sur le marché du travail qu'à l'école, signifiant ainsi qu'ils ont généralement vécu leur scolarisation comme un "échec ». Pour la majorité, leur expérience scolaire négative les a éloignés de l'école dès l'âge de 16 ans. Les jeunes toxicomanes de cette trajectoire se disaient bons travailleurs, rapides et consciencieux. Tous ont rapporté n'avoir rencontré aucune difficulté à se trouver du travail et ont affirmé "savoir se faire valoir ". Ils se sont aussi dits capables d'utiliser leurs réseaux : parents, amis, ressources publiques (centres d'emploi, organismes d'insertion, organismes pour les jeunes).

Malgré cela, à la fin, la consommation les a précipités vers le fond quand elle est devenue persistante, durable et envahissante 
dans tous les domaines de leur vie. À ce moment, les uns ont été congédiés en raison de leur consommation, d'autres ont raté de bonnes possibilités d'emploi à cause d'activités criminelles reliées à leur consommation (p. ex. avoir volé l'employeur). La consommation les a donc conduits petit à petit vers des emplois qu'on peut qualifier de survie: emplois de quelques jours, parfois de quelques heures dépendant des rencontres et des lieux fréquentés. Au moment de l'entrevue, plusieurs n'ont pu formuler de projets professionnels, car ils avaient de la difficulté à identifier leurs intérêts. Plusieurs ont mentionné avoir besoin d'être réorientés sur le plan professionnel.

De plus, l'histoire familiale des jeunes de cette dynamique indique qu'ils ont grandi dans des encadrements familiaux trop rigides, ou trop souples, et peu soutenants à leurs yeux. Plusieurs ont qualifié «d'impossible ou de superficielle » la communication qu'ils avaient avec leurs parents. Certains ont rompu très jeunes avec eux, d'autres désiraient depuis toujours s'en éloigner.

\section{Sylvain, pour faire comme les autres...}

J'ai été adopté lorsque j'étais bébé. Mes parents adoptifs ont bien pris soin de moi, mais ils étaient très sévères, mon père surtout. Parfois, il me frappait. À l'école, j'ai eu bien de la difficulté à me faire accepter. C'est cruel, les enfants. J'avais les cheveux roux, les oreilles décollées et je portais des lunettes. Je faisais rire de moi pas mal. Alors, je me tenais dans mon coin, mais je faisais toutes sortes de bêtises aussi. Je volais mes professeurs et une fois, lorsque j'avais dix ans, j'ai apporté un briquet de la maison et j'ai allumé un feu dans une poubelle. J'ai souvent changé d'école à cause de toutes mes niaiseries et j'ai redoublé deux fois au primaire. Au secondaire, on m'a envoyé dans des classes de troubles du comportement. À 17 ans, j'ai réussi mon secondaire trois et j'ai laissé l'école. J'ai trouvé un premier emploi dans un entrepôt de meubles, par un ami. Je ne consommais pas à ce moment-là. Mais là, j'ai commencé à consommer à chaque pause avec les gars de l'entrepôt. A chaque début et fin de journée, on consommait ensemble. Après cet emploi, j'en ai trouvé beaucoup d'autres. Je trouvais facilement du travail. Si je réussissais à passer une entrevue, j'étais toujours embauché parce je suis un bon travailleur. Je me rappelle que 
«La consommation devient pour les jeunes toxicomanes travailleurs

un mécanisme d'intégration et de reconnaissance dans certains univers professionnels et elle les a précipités vers des emplois de plus en plus précaires. " c'était toujours le même genre d'emplois. Dans ces emplois-là, je trouvais toujours des jeunes avec qui consommer. Tout le monde consommait. Toutes mes paies passaient à acheter du cannabis. J'étais reconnu comme Sylvain le "gars qui paie la traite ". J'étais bien là-dedans, on m'aimait dans ce milieu-là, je ne me sentais pas rejeté. Une fois, j'ai décroché un emploi comme préposé de nuit dans un centre d'alimentation. C'était une job syndiquée. Au moment d'avoir mon attestation syndicale, on m'a surpris à consommer pendant une pause. J'ai été renvoyé, mais ceux qui étaient avec moi, non. C'était injuste, mais je pense que c'est parce que j'étais sur le point d'être syndiqué. Mes parents ont été mis au courant. Ils m'ont mis à la porte, j'avais 20 ans. Je suis allé demeurer chez mon oncle. Présentement, je veux faire une thérapie. Après je vais essayer de voir si je peux retourner à l'école ou me trouver un emploi.

Dans cette dynamique d'identification aux pairs ressort l'importance de s'arrêter au sens de la consommation. On a pu voir que celle-ci est ancrée dans l'histoire individuelle et qu'elle s'est imprimée par la suite dans des cultures de socialisation professionnelles alcoolisantes. La consommation devient pour les jeunes toxicomanes travailleurs un mécanisme d'intégration et de reconnaissance dans certains univers professionnels et elle les a précipités vers des emplois de plus en plus précaires.

\section{Troisième trajectoire : une dynamique de parentification}

Cette trajectoire de précarisation montre que ce qui la sous-tend, c'est l'intégration de rôles et de valeurs inscrites dans l'histoire familiale. En effet, les jeunes toxicomanes de cette trajectoire, illustrée à la figure 4 , se sont retrouvés très tôt dans des contextes les incitant à prendre la place d'un parent et à assumer des responsabilités trop lourdes pour leur âge. Par exemple, ils ont été pourvoyeurs économiques dès la préadolescence ou confidents d'un parent. Ces responsabilités étaient en lien avec leur position d'aîné ou de benjamin dans des familles où la mère assumait seule le rôle de parent. Ce mode de fonctionnement (modèle de conduites et de valeurs) serait à la base de l'enchaînement qui les a 
"En effet, d'un entretien à l'autre, ils ont parlé d'emplois où il $y$ avait trop de travail, trop de tâches à accomplir, trop de problèmes à entendre." maintenus ensuite de façon répétitive dans des emplois où l'écoute et «le don de soi » semblaient aller de soi (p. ex. la coiffure). Bien que le premier emploi ait correspondu à un choix professionnel satisfaisant et souvent en lien avec leur formation, ils l'ont occupé pendant quelques années avant de le quitter pour diverses raisons. En effet, d'un entretien à l'autre, ils ont parlé d'emplois où il y avait trop de travail, trop de tâches à accomplir, trop de problèmes à entendre. À un moment de leur parcours, ils ont dû se retirer du marché du travail pour une période d'au moins un an. Cette période de retrait a constitué pour eux une mise à distance, un refus de continuer à s'impliquer autant dans les domaines privés que professionnels. Cet arrêt a marqué le début d'un sentiment d'impasse professionnelle : « je ne savais pas dans quoi m'en aller ». Néanmoins quand ils ont regagné le marché du travail, ils se sont retrouvés à nouveau dans des emplois similaires (coiffeuse, serveuse dans un bar, aidant dans un organisme de charité), ce qui semble reproduire une spirale de situations auxquelles ils ont été confrontés trop tôt dans leur vie.

Figure 4

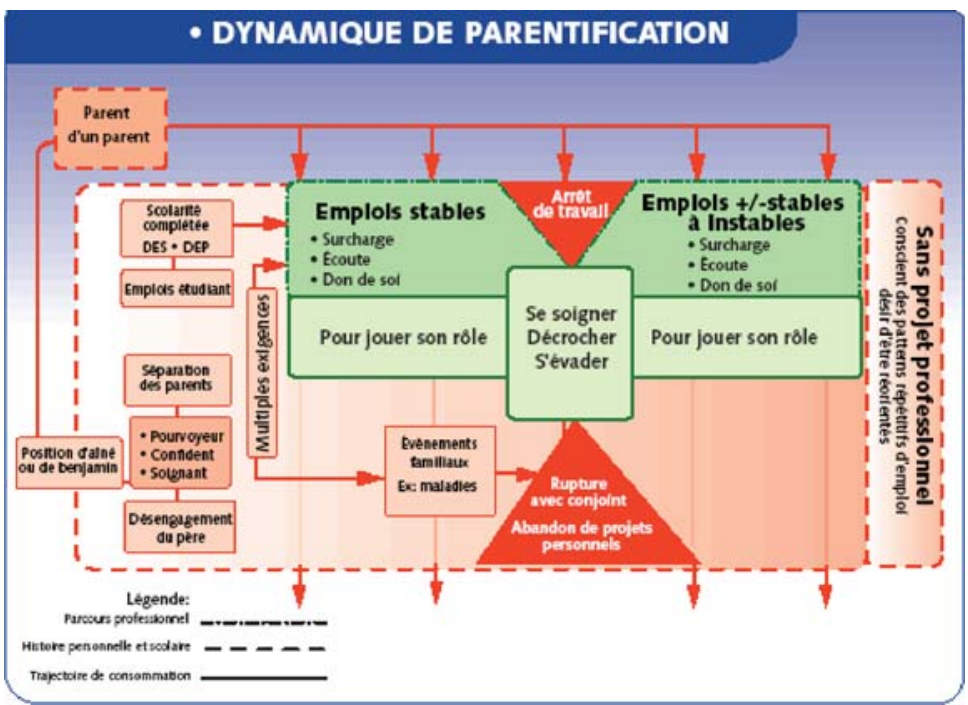


"La consommation d'alcool ou de drogues fut la réponse que ces jeunes ont inventée pour tenir leurs rôles. »
La consommation d'alcool ou de drogues fut la réponse que ces jeunes ont inventée pour tenir leurs rôles. Au moment du déroulement des entretiens, la majorité avait plus de 25 ans, était sans emploi et sans projet professionnel défini. Certains avaient déjà réfléchi aux conséquences du rôle joué et intégré dans l'enfance, et désiraient s'éloigner d'un certain type d'emploi "surtout pas dans le public ».

En somme, l'histoire professionnelle des jeunes de cette trajectoire renvoie à des situations de travail au départ stables mais susceptibles de les rendre vulnérables, jusqu'à l'occupation d'emplois de plus en plus précaires. Dans ce cas-ci, le déclencheur du processus de précarisation est d'avoir joué simultanément des rôles professionnels de la même nature que celle de la vie privée.

\section{Charles, responsable avant l'âge...}

Du plus loin que je me souvienne, j'ai toujours travaillé. C'est moi qui aidais ma mère à payer l'épicerie. Elle élevait seule ses trois enfants et j'étais le plus vieux. Mon père était parti. Tout le long de mon enfance, j'ai regardé en arrière de moi. Il n'y avait personne. C'est moi qui étais en arrière. Encore aujourd'hui, c'est moi qui suis en arrière. Je m'occupe de ma femme et de mes quatre enfants. Les deux plus vieux ne sont pas vraiment les miens, mais ils ne connaissent pas leur père. Moi, je ne les laisserai pas tomber.

À 8 ans j'étais camelot, et à 11 ans, en plus de passer le journal,je livrais pour un dépanneur en bicyclette. Mes journées commençaient à cinq heures le matin et se terminaient à sept heures, le soir. À l'école,j'avais de la difficulté à me concentrer et je faisais bien des coups. J'étais connu par la direction. J'ai tout de même obtenu un diplôme de menuisier.

De 18 ans à 25 ans, mon cousin m'a fait entrer dans une grosse compagnie de vêtements. J'ai commencé comme magasinier puis j'ai eu des promotions. C'était un emploi exigeant et pas payant avec des boss qui me mettaient de la pression. Un moment, $j$ 'ai décroché du système et j'ai retiré du chômage. Pendant à peu près un an, j'ai consommé de l'alcool et des drogues tout le temps. Je suis descendu très bas. Un moment donné, j'ai pensé à mes enfants. Je ne voulais pas qu'ils pensent de moi ce que je pensais de mon père. Là, je suis revenu les pieds sur terre. J'ai trouvé un emploi comme réparateur de 
"Cela signifie que les rôles joués dans la famille, comme enfant, les ont conduits ou contraints à jouer des rôles professionnels semblables. » meubles. C'était un emploi dans ce que j'avais appris. L'entreprise qui m'a engagé ramassait des meubles usagés. Moi, je les réparais et les peinturais. Les meubles étaient vendus pas trop cher. Des fois, j'allais les livrer chez les gens, je voyais que ce monde-là n'était pas très riche. J'avais l'impression d'être utile, mais mon salaire n'était pas bien gros pour faire vivre une famille. Juste un peu au-dessus du salaire minimum et c'était quand même un emploi exigeant. Actuellement, je suis en arrêt de travail parce que j'ai été pris à fumer un joint à l'heure du lunch.

C'est à 15 ans que j'ai commencé à consommer du cannabis et de l'alcool, comme à peu près tout le monde. Cela me faisait relaxer. Après, j'ai consommé du LSD. Avec ça, je pouvais travailler et en même temps m'évader dans ma tête.

L'alcool, la drogue c'est ma petite récompense, c'est mon moyen pour décompresser. Dans ces moments-là, j'ai l'impression que je m'occupe de moi. Parfois, je me dis que j'aimerais suivre un cours en électricité pour être à mon compte et bien faire vivre ma famille. Mais c'est sûr qu'avec quatre enfants, $j$ 'ai des responsabilités, et commencer un autre cours, ce n'est pas évident.

Cette dynamique révèle que le sens d'une trajectoire ne peut être compris en dehors du sujet et de son histoire développementale (Nicole-Drancourt, 1994). En effet, on comprend que c'est dans la continuation des rôles parentaux que les choix professionnels se sont faits. Cela signifie que les rôles joués dans la famille, comme enfant, les ont conduits ou contraints à jouer des rôles professionnels semblables. De là l'importance de relier l'histoire personnelle des individus à leur désir, à leur choix et à leur projet professionnel et d'utiliser des méthodes d'intervention s'appuyant sur la subjectivité et l'approche narrative pour comprendre la dynamique des problèmes qui se posent (Francequin, 2004).

\section{Quatrième trajectoire : une dynamique de compulsion}

Cette trajectoire survient ici de façon inattendue dans un contexte normalement favorable à une intégration professionnelle réussie. Elle a toutefois été enclenchée par un basculement dans la 
cocaïne pour maintenir sa performance corporelle et son image professionnelle. "Devenir rapidement quelqu'un ", dans le sens le plus contemporain du terme, semble le leitmotiv de ces jeunes. Le surinvestissement et l'autoactivation sont aussi des éléments qui forment la trame de cette trajectoire depuis l'entrée des jeunes sur le marché du travail.

En suivant le parcours professionnel des jeunes de cette trajectoire, on peut voir qu'ils se dirigeaient vers des emplois stables, bien rémunérés à leurs yeux et même protégés par une convention collective. Ils ont évoqué des expériences riches et variées (gérant dans la restauration, responsable de projets, superviseur d'équipes dans une industrie de pointe) et de possibilités d'avancement.

Ces jeunes ont commencé leur vie professionnelle avec un capital humain et social significatif : ils avaient un diplôme terminal ou postsecondaire et des relations familiales soutenantes; ils éprouvaient beaucoup de plaisir au travail; ils avaient des relations amicales et faciles avec leurs collègues de travail; ils savaient s'organiser et ils obtenaient une reconnaissance des employeurs. Ils démontraient également un sens des responsabilités au travail, fruit d'un héritage familial bien ancré. Leur vie coulait à grande vitesse et leur rythme de travail semblait sans limites multipliant les heures, les emplois et les sorties récréatives. Comme l'exprime Hautefeuille (2004, p. 9), "une vie quotidienne devenue un parcours de combattant». La figure 5 illustre cette dynamique de compulsion.

Les jeunes toxicomanes de cette trajectoire ont laissé voir que dès le début de leur parcours professionnel ils consommaient abusivement de l'alcool, ensuite de la cocaïne qui semblait mieux convenir aux valeurs de performance et d'intensité qui les attiraient. Ils ont aussi découvert que la cocaïne est une drogue qui leur permettait mieux que l'alcool de maintenir leur image sociale et leur productivité, et qui s'apparentait à la pharmacologie de l'adaptation du paraitre et de la normalisation (Hautefeuille, 2004). Selon leurs propos, conserver son image signifiait consommer pour durer plus longtemps : pour ne pas dormir, pour ressentir plus longtemps les effets bénéfiques de la drogue, sans les effets apparents de l'ébriété, pour performer dans la compétition. Cependant, après quelques 
années de consommation, certaines pressions (c.-à-d. vols ou fraudes chez les employeurs, mauvaises performances, accidents, rupture amoureuse) les ont obligés à des prises de conscience face à leur consommation. En effet, au moment où tout a basculé, ils étaient dans une double situation de précarité :précarité financière en raison de nombreuses dettes contractées dans des épisodes de défonce, et précarité de consommation parce qu'ils en ont perdu le contrôle. Au moment de l'entrevue, ils tentaient de régler leur problème de consommation et ils voulaient poursuivre leur carrière professionnelle.

Figure 5

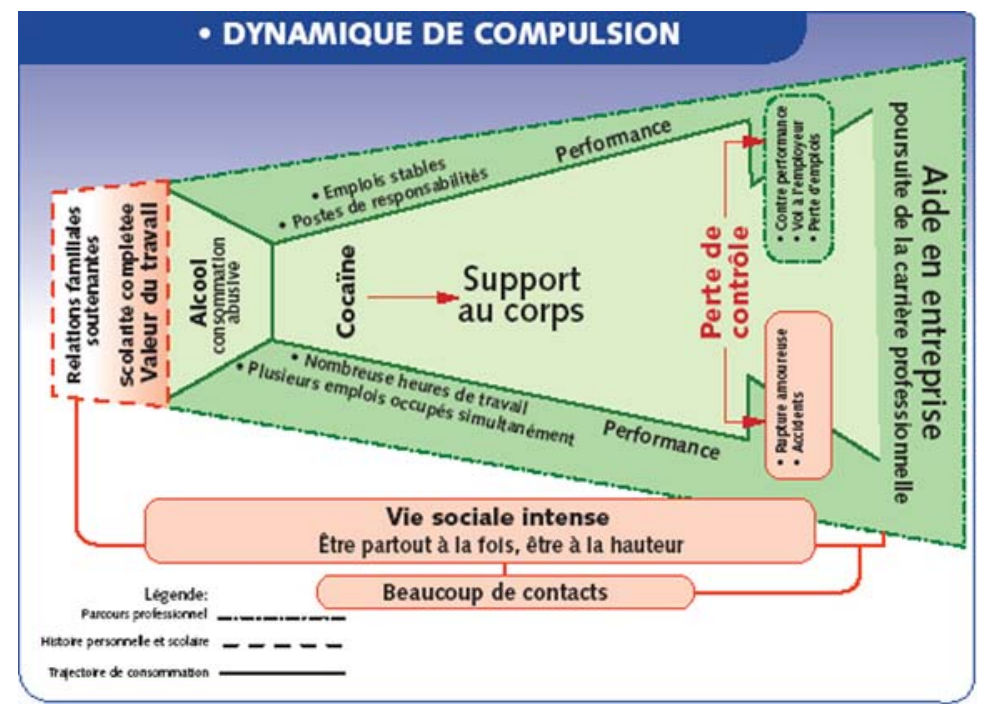

\section{Jonathan, un gars sans limites...}

À treize ans, j'ai commencé à boire de la bière, c'était commun dans mon coin, c'était comme normal, mais quand même je buvais plus que les autres. C'était toujours un petit peu plus que les autres pour tout. Il fallait toujours que je sois le meilleur. J'allais à l'école et je travaillais déjà parce que j'avais besoin d'argent pour m'acheter toutes sortes d'affaires que les jeunes aiment à cet âge-là, par exemple une bicyclette. Dans ma famille, 
"Paradoxalement, les milieux de travail peuvent rendre des jeunes travailleurs passionnés et ambitieux, à risque de succomber aux attraits de l'hyperactivité et de développer une dépendance à la toxicomanie..." le travail est quelque chose d'important. Mes parents m'encourageaient beaucoup à travailler, mais il fallait que je poursuive mes études en même temps. Ils étaient fiers de moi.J'étais considéré comme un jeune capable, entreprenant et responsable. J'étais sociable aussi. Dans mon coin, j'étais connu. Je travaillais pour plusieurs employeurs en même temps. J'ai tout de même eu mon diplôme au CEGEP.

À 23 ans, j'ai trouvé un emploi dans mon domaine. Je travaillais beaucoup. J'étais payé pour 40 heures par semaine, mais j'en faisais jusqu'à 60. Je voulais organiser un nouveau système informatique pour la compagnie qui m'avait embauché. Je m'investissais à plein. Plus mes patrons me voyaient aller, plus ils avaient des projets pour moi.Je mettais de l'argent de côté, car j'allais me marier et acheter une maison. À cette époque,j'étais "sur le party" pas mal. Je sortais beaucoup et je fermais souvent les bars. Je vivais à cent milles à l'heure et là j'ai commencé à toucher à la coke. Avec l'alcool, j'étais rendu épuisé, fatigué, je manquais d'énergie, j'avais des brûlements d'estomac, cela me prenait une journée à m'en remettre. Avec la coke, le lendemain, j'étais en forme et j'avais de l'énergie à revendre. En plus, j'étais moins étiqueté alcoolique, j'étais moins étiqueté "un gars de party". Mais consommer de la coke, ça me coûtait beaucoup plus cher. J'ai dépensé mes économies, j'ai rempli mes cartes de crédit et je me suis mis à emprunter à gauche et à droite. Puis, ma blonde m'a quitté,j'étais plus vivable. À 25 ans, j'avais tellement de problèmes personnels que mon travail a commencé à s'en ressentir, puis j'ai eu un accident de voiture. J'ai dû déclarer faillite et j'ai avoué à mes parents que j'étais toxicomane et que je devais suivre une thérapie. C'est sûr que je les ai déçus, mais ils m'ont supporté. Avec eux, je vais avoir l'aide qu'il faut pour me relever et continuer.

Cette dynamique de compulsion n'est pas sans rappeler celle qui prévaut dans certains milieux de travail. Ces dernières décennies en effet, l'intensification du travail s'est traduite par une stratégie efficace du management afin d'obtenir plus de productivité et de performance de la part du personnel. Paradoxalement, les milieux de travail peuvent rendre des jeunes travailleurs passionnés et ambitieux, à risque de succomber aux attraits de l'hyperactivité et de développer une dépendance à la toxicomanie (Maranda, Morissette et Lessard, 2002). 


\section{Conclusion}

Selon nos résultats, la réalité de la précarisation du travail chez les jeunes ayant vécu une expérience de toxicomanie est complexe. Elle résulte de dynamiques à la croisée de blessures significatives à plusieurs dimensions : individuelles, familiales ou professionnelles. Notre recherche a fait état de quatre dynamiques de précarisation qui sous-tendent les trajectoires socioprofessionnelles de jeunes consommateurs âgés de 18 à 30 ans : d'infériorisation, d'identification, de parentification et de compulsion.

Dans le cas de la dynamique d'infériorisation, une enfance abusée, couplée à des emplois abusifs qui exploitent le corps de ces jeunes devenus toxicomanes, a fait en sorte que ces derniers ont eu recours aux drogues pour oublier une vie déjà trop difficile. On reconnaît là le sort de bien des personnes qui, de la famille au monde du travail, se retrouvent dans des situations similaires, où est réactivée une dramatique existentielle de l'histoire de vie. En effet, certaines situations de travail font ressurgir une souffrance héritée du passé (Maranda et collab., 2006). Ainsi, les stratégies défensives de ces jeunes furent de se soumettre à des contextes abusifs qui se sont renforcés et la drogue les a confinés dans une posture de victime.

En ce qui concerne la dynamique d'identification, ces jeunes ont d'abord vécu des situations de rejet, dont l'échec scolaire, et se sont retrouvés par la suite dans des petits boulots ou des emplois de survie. Ils sont entrés dans un cercle vicieux. En raison de leur signe distinctif, ils ont commencé à consommer pour socialiser, pour s'intégrer, pour faire partie du groupe. Dans les milieux où ils ont travaillé, existe souvent une culture de consommation d'alcool compensant des conditions pénibles de travail (Maranda, 1998). Comme le disent Dejours et Burlot (1985), l'alcool et les drogues sont de puissants anxiolytiques. La consommation d'alcool et de drogues au travail ou autour du travail dans les bars avoisinants, fait partie de cette idéologie défensive destinée à tromper diverses émotions associées à ce dernier. "L'alcool facilite la reprise du travail, il désinhibe les défenses individuelles contre le travail 
"Pour mettre à jour le processus d'alcoolisation on de toxicomanie des personnes, il faut, à notre avis, étudier conjointement le monde structurel et le monde individuel, puis comprendre le sens de l'utilisation de l'alcool ou des drogues. » dangereux » (p. 108). Par conséquent, il ne faudrait pas négliger les incidences du travail dans la compréhension du développement de la toxicomanie. Les jeunes de cette catégorie, qui avaient un grand besoin de se faire accepter de leur groupe de travail, ont été socialisés dans cette culture du recours aux psychotropes pour se récompenser, se calmer ou se donner du courage. Ils font partie de ceux qui sont devenus dépendants de ces produits.

La troisième dynamique, parentification, illustre le cas de jeunes qui ont dû assumer des responsabilités parentales qui ne leur revenaient pas. Une fois devenus jeunes adultes, « naturellement », ils ont occupé le même type d'emploi. Ils ont craqué sous le poids des malheurs d'autrui et pour tenir leur rôle, ou le coup, ils ont utilisé de l'alcool ou des drogues en guise d'automédication (Legrand, 2008 et 1997). Pour atténuer une souffrance inacceptable, ces jeunes ont trouvé dans l'alcool ou les drogues « une solution à un problème "; cependant, le moyen est devenu la finalité et ils sont tombés dans un piège circulaire : boire pour boire, consommer des drogues pour leurs effets, semblables à ceux des médicaments.

Finalement, une dynamique de compulsion montre la situation de jeunes aux ambitions élevées qui s'inscrivent dans des conduites extrêmes construites sous le mode de l'hyperactivité. Pour faire face à une organisation du travail, elle-même construite sous le mode de l'hypertravail, ils ont utilisé des drogues pour augmenter leur performance. La tentation a été grande pour ces jeunes d'utiliser des adjuvants afin de faire face à la compétitivité.Voilà ici le piège des stratégies défensives : elles se retournent contre le sujet. En ce sens, les jeunes fortement sollicités par les valeurs de performance sont plus à risque qu'on ne le croit.

En somme, la précarisation est à la fois l'explication et le résultat de situations qui se renforcent. Pour mettre à jour le processus d'alcoolisation ou de toxicomanie des personnes, il faut, à notre avis, étudier conjointement le monde structurel et le monde individuel, puis comprendre le sens de l'utilisation de l'alcool ou des drogues.

En premier lieu, il s'avère important d'étudier ces problèmes en lien avec les contextes dans lesquels ils surviennent et se 
«En ce sens, la clinique et l'utilisation des méthodes qui mettent en lien la situation actuelle (synchronique) avec l'histoire des personnes (diachronique) sont porteuses d'analyses qui dépassent les explications comportementales ou même cognitives. ” développent. Pour y intervenir, il faut d'abord comprendre la dynamique complexe des problèmes en présence et développer des stratégies à plusieurs paliers. Notre recherche démontre que le contexte structurel d'emplois précaires a joué dans l'apparition ou le développement de l'alcoolisme ou des toxicomanies de ces jeunes. En effet, les effets structurels du marché du travail et organisationnels des pratiques managériales, ainsi que les modes de socialisation des milieux de travail, puis les pratiques de consommation de ces jeunes se sont combinés pour devenir des facteurs de risques de toxicomanie.

Cette réalité plurielle met de l'avant l'importance de concevoir des interventions compréhensives tenant compte des dynamiques de consommation. En ce sens, la clinique et l'utilisation des méthodes qui mettent en lien la situation actuelle (synchronique) avec l'histoire des personnes (diachronique) sont porteuses d'analyses qui dépassent les explications comportementales ou même cognitives. En effet, les récits de vie, ou récits de pratique, donnent la parole aux sujets. Ce sont ces derniers qui sont en mesure de raconter la suite des événements ou incidents qui ont jalonné leur vie et de donner un sens aux expériences vécues. Des outils de recherche et d'intervention ont été conçus par les chercheurs qui développent ces approches, notamment en sociologie clinique, et s'avèrent des pistes prometteuses pour intervenir "autrement ", c'est-à-dire en redonnant la possibilité aux sujets de s'approprier ou de se réapproprier quelque chose de leur propre histoire (Maranda et collab., 2007). De ces prises de conscience, qui donnent du sens aux trajectoires empruntées, et de la compréhension qui s'en dégage même si elle peut être parfois dérangeante, il est possible ensuite de se mettre en action, ou de se remettre en action, comme sujet de sa propre histoire (de Gaulejac, 1999).

Un ensemble d'acteurs sociaux sont concernés par l'apparition ou le développement de la toxicomanie (patronat, syndicats, milieux scolaires, intervenants) puisque certaines difficultés découlant d'éléments du contexte échappent au seul contrôle des personnes. Les entreprises sont de plus en plus interpellées dans cette dynamique (INRS, 2008), notamment en raison des dangers 
"Il s'agira d'intervenir pas seulement $d u$ côté des personnes aux prises avec cette problématique, mais aussi du côté des milieux qui les emploient. » que représente l'utilisation de substances psychoactives légales ou illicites. Des enjeux politiques, économiques et personnels sont en présence (Fontaine, 2004). Un rôle accru et concerté de ces différents acteurs (Morissette, Maranda et De Montigny, 1997) apparaît nécessaire sur deux plans : celui de l'insertion (ou de la réinsertion) professionnelle (Morissette, Maranda et Lessard, 2006) et celui du maintien des jeunes dans les milieux de travail. L'intervention consiste donc à prendre en compte de façon synchronique : des éléments du contexte professionnel et personnel, le sens des conduites humaines, et la compréhension ou l'analyse de stratégies d'adaptation ou défensives en présence. De plus, du côté de la prévention primaire des facteurs de risque en milieu de travail, beaucoup reste à faire pour identifier les situations à risque de toxicomanie ou de rechute. Il s'agira d'intervenir pas seulement du côté des personnes aux prises avec cette problématique, mais aussi du côté des milieux qui les emploient. Le comportement humain n'étant pas réductible à des éléments objectifs, il faut donc donner la parole aux sujets pour comprendre le sens qu'ils attribuent à leurs expériences, à leur cheminement et à leurs comportements avant de les remettre sur le marché du travail. Un accord de sens entre les personnes concernées et les intervenants permettrait d'éviter la reproduction de situations d'échec. Ce type d'accord doit aussi être entamé avec les milieux de travail et demande une participation compréhensive des employeurs. Pour cela, il faut que le dialogue s'installe entre les différents intervenants interpellés dans l'insertion (ou la réinsertion) socioprofessionnelle, y compris ceux du monde du travail, et de ce côté, des efforts prodigieux doivent être accomplis (Negura et Maranda, 2008). Il y va de l'avenir d'une jeune génération de travailleurs dont les pratiques de consommation de produits sont bien différentes de celles de leurs aînés.

\section{Bibliographie}

AMES, Genevieve M.,JoelW.GRUBE et Rolland S. MOORE (2000). « Social control and workplace drinking norms: A comparison of two organizational cultures ", Journal of Studies on Alcohol, 61(2), p. 203-219. 
APPAY, Béatrice (2005). La dictature du succès, le paradoxe de l'autonomie contrôlée et de la précarisation, L'harmattan, Logiques sociales.

APPAY, Béatrice, et Annie THÉBAUD-MONEY (INSERM), (1997). Précarisation sociale, travail et santé, IRESCO/CNRS.

BERNIER, Jean, Guylaine VALLÉE et Carol JOBIN (2003). Les besoins de protection sociale des personnes en situation de travail non traditionnelle, Synthèse du Rapport final, Gouvernement du Québec, Ministère du Travail, 42 p.

BERTAUX, Daniel (1997). Les récits de vie, Paris, Nathan.

BRAY, Jeremy W., et collab. (2000). "Symptoms of dependence, use, and labour market outcomes ", American Journal of Drug and Alcohol Abuse, 26(1), p. 77-95.

CASTEL, Robert (2007). "Au-delà du salariat ou en deçà de l'emploi? L'institutionnalisation du précariat?", sous la direction de Serge Pangam, Repenser la solidarité : l'apport des sciences sociales, Paris, PUF (Collection « Le lien social»), p. 416-433.

CASTEL, Robert (1998). Les sorties de la toxicomanie, Suisse, Éditions Universitaires Fribourg.

COOK, Royer, et William SCHLENGER (2002). "Prevention of substance abuse in the workplace: Review of research on the delivery of services ", The Journal of Primary Prevention, 23(1), p. $115-142$.

CRANFORD, Cynthia J., Leah F.VOSKO et Nancy ZUKEWICH (2003). « Precarious employment in the canadian labour market: a statistical portrait " Just Labour, 3, p. 6-22.

DAMBUYANT-WARGNY, Gisèle (2006). Quand on n'a plus que son corps, Paris, Armand Colin.

DAMBUYANT-WAR GNY, Gisèle (2000). Entre choix et destin, la condition sociale des plus démunis. La place du corps dans la désocialisation, Thèse de doctorat en sociologie, E.H.S.S., Paris.

DE GAULEJAC,Vincent (1999). L'histoire en héritage : roman familial et trajectoire sociale, Paris, Desclée de Brouwer.

DEJOURS, Christophe, et A. BURLOT (1985). Contribution de la psychopathologie du travail à l'étude de l'alcoolisme. Psychopathologie du travail, sous la direction de C. Dejours, C.Veil et A. Wisner, Entreprise moderne d'édition, p. 105-11.

FACY, Françoise, et E. LE HUEDE (1992). Étude épidémiologique des centres de post-cure 1989-1991, France, Institut national de la santé et de la recherche médicale (INSERM).

FONTAINE, Astrid (2004). Drogues et travail, des enjeux politiques, économiques et personnels, Revue toxibase, Drogues et travail : le new deal?, 15, 3e trimestre, p. 1-4.

FRANCEQUIN, Ginette (2004). Pour une approche biographique en orientation :l'intégration de l'histoire personnelle au choix de profession, Québec, Éditions Septembre.

FRONE, Michael R. (2003). «Predictors of Overall and On-the-Job Substance Use Among Young Workers " Journal of Occupational Health Psychology, 8(1), p. 39-54.

FRONE, Michael R. (1999). "Work stress and alcohol use ", Alcohol Research and Health, 23, p. 284-291.

HAUTEFEUILLE, Michel (2004). "Drogues et travail, des enjeux politiques, économiques et personnels ", dans Drogue et monde du travail : le dopage au quotidien, Revue Toxibase,15(3), p. 9-11.

HOWARD, Anne (1995). The Changing nature of work, San Francisco, CA: Jossey-Bass. 
INSTITUT NATIONAL DE RECHERCHE ET DE SÉCURITÉ (2008). La lettre d'information, Dossier web Addictions et travail, mars.

IVERSEN, Roberta Rehner (1998). "Occupational social work for the 21st century » Social Work, 43(6), p. 551-566.

JAMOULLE, Pascalle (2000). Drogues de rue. Récits et styles de vie, Bruxelles, De Boeck University.

LAFLAMME, Claude (2000). La poursuite des études : une question de pouvoir du diplôme et de marginalisation. Les 18 à 30 ans et le marché du travail, quand la marge devient norme, sous la direction de G. Fournier et B. Bourassa, CRIEVAT, Les Presses de l’Université Laval, p. 119-138.

LANDRY, Serge, Louise GUYON et Brigitte BEAUVAIS (1997). Évolution du profil biopsychosocial de la clientèle toxicomane en réadaptation à Domrémy-Montréal entre 1991-1995, Montréal, Recherche et intervention sur les substances psychoactives-Québec.

LEGRAND, Michel (2008). D'une grande histoire de vie, une aventure singulière. Intervenir par le récit de vie, sous la direction de Vincent de Gaulejac et Michel Legrand, Sociologie clinique, Érès, p. 195-212.

LEGRAND, Michel (1997). Le sujet alcoolique, essai de psychologie dramatique, Paris : Desclée de Brouwer, $258 \mathrm{p}$.

LEGRAND, Michel (1993). L'approche biographique: théorie, clinique, Marseille, Hommes et perspectives.

LOPEZ, Dominique (2002). "Consommation de drogues illicites et exclusion sociale : état des connaissances en France », Tendances, OFDT, 24, Disponible à l'adresse suivante http://wwww. drogues.gouv.fr.

MATHIS, Diane A., et collab. (1994). "Service needs of injection drug users : Gender and racial differences ", dans R.R. Watson (Ed.), Alcohol and Drug Abuse Reviews (vol.14, 329-358), Clifton, NJ: Humana.

MARANDA, Marie-France, et collab. (2007). Récit de vie et psychodynamique du travail : étude sur la toxicomanie en milieu de travail. Récits de vie et sociologie clinique, sous la direction de L. Mercier et J. Rhéaume, IQRC, Collection Culture et société, p. 277-300.

MARANDA, Marie-France, Jacques RHÉAUME et Christian GENEST (2006). Toxicomanie : histoire de vie, histoire de travail, une seule trame. Espace de réflexion, espace d'action en santé mentale au travail, Enquêtes en psychodynamique du travail au Québec, sous la direction de l'Institut de psychodynamique du travail du Québec, PUL, p. 169-193.

MARANDA, Marie-France (1998). "Compromis fordiste et individualisation des stratégies de défense ", Stress, santé et intervention au travail, Collection Gestion des paradoxes dans les organisations, tome 7, sous la direction de R. Jacob et R. Laflamme, p. 151-158.

MARANDA, Marie-France, Pauline MORISSETTE et Danielle LESSARD (2002) "Précarité du travail et toxicomanie : un parcours socioprofessionnel doublement fragilisé ", Pratiques psychologiques, 4, p. 109-122.

MORISSETTE, Pauline, et Denise BOIVIN (1999). Rapport de recherche sur la réalité socioprofessionnelle des toxicomanes dans les centres publics québécois, Montréal, CQRS.

MORISSETTE, Pauline, Marie-France MARANDA et Marie-Josée DE MONTIGNY (1997). Prévention de l'abus de substances psychoactives et milieu de travail : portrait et analyse de pratiques québécoises, Rapport de recherche, Comité permanent de lutte aux toxicomanies, Ministère de la Santé et des Services sociaux, Gouvernement du Québec. 
MORISSETTE, Pauline, Marie-France MARANDA et Danielle LESSARD (2006). "Young Substance Abusers and precarious employment:Trajectories and Anchoring Dynamics » Canadian Journal of Counselling, 40(3), p. 175-191.

MUCCHIELLI, Alex (1996). Dictionnaire des méthodes qualitatives en sciences humaines et sociales, Paris, Armand Collin.

NEGURA, Lilian, et Marie-France MARANDA (2008). « Hiring Substance Abusers: Attitudes of Managers and Organizational Needs ", Drugs: Education, Prevention \& Policy, 15 (2), p. 129-144.

NICOLE-DRANCOURT, Chantal (1994). "Mesurer l'insertion professionnelle ", Revue française sociologique, XXXV, p. 37-68.

OBSERVATOIRE FRANÇAIS DES DROGUES ET DES TOXICOMANIES (2002). Drogues et dépendances. Indicateurs et tendances 2002, Paris, rapport OFDT.

PAUGAM, Serge (2000). Le salarié de la précarité, Paris, Les Presses universitaires de France.

PLATT, Jerome J. (1997). "Vocational Rehabilitation of Drug Abusers », dans G.A. Marlatt, G.R. Vanden Bos \& R. Gary (Eds.), Addictive Behaviors: Readings on Etiology, Prevention, and Treatment (p.759-801). Washington, DC:American Psychological Association.

POLLERT, Anna (1988). «The "flexible firm": Fixation or fact?", Work, Employment and Society, 2(3), p. 281-316.

PURCELL, Kate, et John PURCELL (1998). "In-sourcing, out-sourcing, and the growth of contingent labour as evidence of flexible employment strategies ", European Journal of Work and Organizational Psychology, 7, p. 39-59.

ROMAN, Paul M., et Terry C. BLUM (1999). "Externalization and internalization as frames for understanding workplace deviance. The management of alcohol and drug abuse ", Research in the Sociology of Work, 8, p. 139-164.

ROSE, José (1998). Les jeunes face à l'emploi, Sociologie Économique, Paris, Desclée de Brouwer.

ROSE, José (2000). "Les jeunes et l'emploi : questions conceptuelles et méthodologiques ", dans G. Fournier et B. Bourassa «éd. » Les 18 à 30 ans et le marché du travail, Ste-Foy, Les Presses de l'Université Laval, p. 83-116.

SAINT-MARTIN, Corinne (2007). "Précarisation des populations urbaines, fragilisation professionnelle des travailleurs sociaux », Empan, 68, p. 52-57.

SKINNER, Harvey A. (1982). "The drug abuse screening test », Addictive Behaviors, 7(4), p. 363-371.

STRAUSS, Anselm, et Juliet CORBIN (1998). Basics of qualitative research: Techniques and procedures for developing grounded theory, Thousand Oaks, Calif. : Sage Publications.

\section{Notes}

1. Cet article est dédié à la mémoire de Michel Legrand, décédé en 2006. Michel a agi pour nous comme un mentor dans l'étude de la méthodologie des récits de vie, en lien avec l'alcoolisme et la toxicomanie. Il nous a accordé beaucoup de temps et de considération. Nous désirons souligner son importante contribution dans le domaine de la toxicomanie et inviter les lecteurs à prendre connaissance de ses écrits.

2. Cette recherche a été réalisée à l'aide d'une subvention du Conseil québécois de la recherche sociale (CQRS). 